Frau Prof. Dr. med. C. Bayerl im Gespräch mit Herrn Prof. Dr. med. Hans Meffert:

\section{MTX-Behandlung bei Psoriasis - Was muss bedacht werden?}

C. Bayerl: Lieber Herr Professor Meffert, Sie haben die Seite gewechselt, sich in unsere Patienten versetzt und die Fachinformation Methotrexat gelesen. Was war Ihnen dabei aufgefallen?

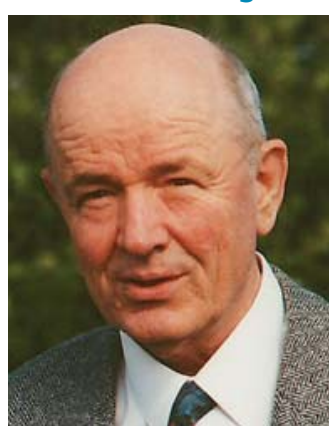

H. Meffert: Richtig indiziert und angewandt gilt vielen Kollegen, wie auch mir, Methotrexat (MTX) als probates Mittel gegen Psoriasis. Auch die Kombination einiger Biologika und MTX ist andernorts zugelassen. Seit ich die aktuelle Fachinformation über Methotrexat „Lederle“ Tabletten gelesen habe, grübele ich darüber nach, warum dort nicht weniger als siebzehnmal Worte wie Tod, tödlich oder Todesfälle vorkommen. Weiß jemand warum? Wohlgemerkt, diese Todesmahnungen gelten auch für die moderate Dosierung bei Psoriasis, beispielsweise $15 \mathrm{mg}$ einmal wöchentlich.

C. Bayerl: Ursprünglich zur Krebsbehandlung zugelassen, ist MTX definitionsgemäß ein zytotoxisches Medikament. Wie ist die Studienlage?

H. Meffert: MTX wird seit mehr als 60 Jahren zur Psoriasisbehandlung genutzt. Damals waren die Preise für neu eingeführte Medikamente moderat und heutzutage geforderte Studien so gut wie nicht finanzierbar. Jedoch wusste man über mögliche unerwünschte Wirkungen von Anfang an gut Bescheid. So besprach Walter Lever bereits in seinem Artikel „Die Behandlung der Psoriasis mit Methotrexat" (Dermatol Monatsschr 1970; 156: 493 -497) wesentliche Fakten und Erfahrungen. Im Einzelnen wurden u.a. angeführt

- Unterdrückung der Hämatopoese

- Die toxische Wirkungen von MTX auf die Leber

- Lebererkrankungen und aktive Magen- oder Duodenalulzera als Kontraindikationen

- Leberdiagnostik: Enzyme und ggf. perkutane Nadelbiopsie

- Weitere Enzymuntersuchungen

- Nierenfunktionsprüfungen bei Nierenerkrankungen in der Vorgeschichte

- Multiple aphthenartige Ulzerationen im Munde

- Empfängnisverhütung ab drei Monate vor möglicher Konzeption. MTX einnehmende Männer sollten davor gewarnt werden, Nachkommenschaft zu zeugen

- Ob die MTX-Behandlung über längere Zeit durchgeführt werden könne, sei insbesondere mit Rücksicht auf mögliche irreversible Leberschäden fraglich.

C. Bayerl: Und wie steht es jetzt um diesen möglichen irreversiblen Leberschaden?

H. Meffert: Nicht zuletzt dank Walter Lever wissen wir jetzt viel mehr darüber. Letzterer stammte aus Erfurt und besuchte dort in den Sechziger und Siebziger Jahren mehrfach seine Mutter und seinen dort als Hautarzt praktizierenden Bruder Fritz. Dann hielt er regelmäßig auch einen Vortrag in der Hautklinik der Medizinischen Akademie Erfurt. Dorthin pilgerten große Teile der Thürin- ger Dermatologenschaft, staunten über Neuerungen aus der großen weiten Welt und übernahmen die MTX-Behandlung der Psoriasis.

Jahrzehnte später konnte U. Wollina die Daten der Jenaer Universitätshautklinik auswerten. Unter der Überschrift „Toxicity of methotrexate treatment in psoriasis and psoriatic arthritis short- and long-term toxicity in 104 patients" (Clin Rheumatol 2001; 20: 406-410) stellte er fest, dass Leberveränderungen und Anstiege der Enzymaktivitäten bei den MTX-Behandelten nicht signifikant häufiger als in der Kontrollgruppe vorkamen. Unerwünschte Arzneimittelreaktionen seien unter MTX-Therapie häufig, meist mild, vorübergehend und von der Dosis unabhängig.

C. Bayerl: Wie gehen Sie in der Sprechstunde in Zukunft vor? H. Meffert: Zunächst sollte man die aktuelle S3-Leitlinie Psoriasis durchforsten. Übrigens kommt dort im Zusammenhang mit MTX das Wort Tod nur einmal vor. Unerlässlich ist auch die Kenntnis der einschlägigen Fachinformationen. Niemals sollten die in der aktuellen S3-Leitlinie angeführten wesentlichen Gesichtspunkte unberücksichtigt bleiben

- Kontrollparameter: Blutbild (Hb, Hkt, Differenzialblutbild, Thrombozyten), Nierenfunktion (Serumkreatinin, Harnstoff, Urinsediment), Leberwerte (Serumtransaminasen), aminoterminales Propeptid von Typ III-Prokollagen

- Wesentliche Gegenanzeigen: Leberfunktionsstörungen, Schwangerschaft

- Wesentliche unerwünschte Arzneimittelwirkungen: Leberfibrose und -zirrhose, Pneumonie/Alveolitis, Knochenmarksdepression, Nierenschädigung.

Obligate Laboruntersuchungen sind fällig

- Vor Therapiebeginn: Komplettes Blutbild mit Differenzialblutbild und Thrombozyten, Leberenzyme (ALAT [GPT], ASAT [GOT], AP), Bilirubin, Serumalbumin und Nierenretentionsparameter (Kreatinin, ggf. Kreatinin-Clearance). Hepatitis-Serologie (A, B, C), ggf. Tuberkulose-Ausschluss (aminoterminales Propeptid von Typ III-Prokollagen), ggf. Thorax-Röntgen

- Während der Therapie: In den ersten beiden Wochen wöchentlich, dann zweiwöchentlich für den nächsten Monat. Danach, abhängig von der Leukozytenzahl und der Stabilität des Patienten, etwa monatlich

\section{Dosierung}

Empfohlene Initialdosis (bezogen auf einen durchschnittlichen Erwachsenen von $70 \mathrm{~kg}$ Körpergewicht): Einmalige Testdosis von 2,5 bis $5 \mathrm{mg}$ zur Abschätzung der Toxizität. Bei unveränderten Laborparametern eine Woche später Fortführung mit etwa $7,5 \mathrm{mg}$. Die Dosis wird unter Überwachung der Laborparameter schrittweise (in Schritten von 5 - 7,5 mg pro Woche) auf üblicherweise $15 \mathrm{mg}$ gesteigert.

\section{Gegenanzeigen}

- Überempfindlichkeit gegen den Wirkstoff MTX oder sonstige Bestandteile

- Nierenfunktionsstörungen (Kreatinin-Clearance kleiner $60 \mathrm{ml} / \mathrm{min}$ )

- Leberschaden

- Erkrankung des blutbildenden Systems

- Erhöhter Alkoholkonsum 
Immundefizienz

- Schwere Infektion

- Ulzera des Magen-Darm-Traktes

\section{Ausgewählte besondere Warnhinweise und} Vorsichtsmaßnahmen

- Keine zusätzlich leberschädigenden Arzneimittel einnehmen, auf Alkohol verzichten bzw. den Alkoholkonsum deutlich einschränken

- Erbrechen, Diarrhö, Stomatitis können die Toxizität von MTX steigern; Anwendung dann unterbrechen

- Besondere Vorsicht bei insulinpflichtigem Diabetes mellitus sowie bei Einschränkung der Lungenfunktion

- Keine Impfungen mit Lebendimpfstoff

- Bei inaktiver chronischer Infektion (z. B. Zoster, Tuberkulose, Hepatitis B oder C) ist deren Aktivierung möglich

- Insbesondere ältere Patienten in kurzen zeitlichen Abständen auf frühe Zeichen von Toxizität untersuchen

- Männer, die mit MTX behandelt werden, sollten während der Behandlung und 6 Monate danach kein Kind zeugen und sich vor Therapiebeginn wegen der Möglichkeit schwerwiegender Störungen der Spermatogenese durch die Therapie über eine Spermakonservierung beraten lassen. Frauen sollten während der MTX-Therapie nicht schwanger werden.

Schließlich kann man im schlimmsten Falle nur hoffen, dass auch Staatsanwalt und Richter die neue S3-Leitlinie gebührend berücksichtigen.

Prof. Dr. med. Hans Meffert

Dermatologisches Zentrum Berlin

Potsdamer Chaussee 80

14129 Berlin-Nikolassee

hans.meffert@web.de 